\title{
MRI Tractography of Corticospinal Tract and Arcuate Fasciculus in High-Grade Gliomas Performed by Constrained Spherical Deconvolution: Qualitative and Quantitative Analysis
}

\author{
E. Mormina, M. Longo, A. Arrigo, C. Alafaci, F. Tomasello, A. Calamuneri, S. Marino, M. Gaeta, S.L. Vinci, and F. Granata
}

\begin{abstract}
BACKGROUND AND PURPOSE: MR imaging tractography is increasingly used to perform noninvasive presurgical planning for brain gliomas. Recently, constrained spherical deconvolution tractography was shown to overcome several limitations of commonly used DTI tractography. The purpose of our study was to evaluate WM tract alterations of both the corticospinal tract and arcuate fasciculus in patients with high-grade gliomas, through qualitative and quantitative analysis of probabilistic constrained spherical deconvolution tractography, to perform reliable presurgical planning.
\end{abstract}

MATERIALS AND METHODS: Twenty patients with frontoparietal high-grade gliomas were recruited and evaluated by using a $3 T$ MR imaging scanner with both morphologic and diffusion sequences (60 diffusion directions). We performed probabilistic constrained spherical deconvolution tractography and tract quantification following diffusion tensor parameters: fractional anisotropy; mean diffusivity; linear, planar, and spherical coefficients.

RESULTS: In all patients, we obtained tractographic reconstructions of the medial and lateral portions of the corticospinal tract and arcuate fasciculus, both on the glioma-affected and nonaffected sides of the brain. The affected lateral corticospinal tract and the arcuate fasciculus showed decreased fractional anisotropy $(z=2.51, n=20, P=.006 ; z=2.52, n=20, P=.006)$ and linear coefficient $(z=2.51$, $n=20, P=.006 ; z=2.52, n=20, P=.006)$ along with increased spherical coefficient $(z=-2.51, n=20, P=.006 ; z=-2.52, n=20, P=$ .006). Mean diffusivity values were increased only in the lateral corticospinal tract $(z=-2.53, n=20, P=.006)$.

CONCLUSIONS: In this study, we demonstrated that probabilistic constrained spherical deconvolution can provide essential qualitative and quantitative information in presurgical planning, which was not otherwise achievable with DTI. These findings can have important implications for the surgical approach and postoperative outcome in patients with glioma.

ABBREVIATIONS: $\mathrm{AF}=$ arcuate fasciculus; $\mathrm{Cl}=$ linear coefficient; $\mathrm{Cp}=$ planar coefficient; $\mathrm{Cs}$ = spherical coefficient; $\mathrm{CSD}$ = constrained spherical deconvolution; $\mathrm{CST}=$ corticospinal tract; $\mathrm{FA}=$ fractional anisotropy; $\mathrm{HGG}=$ high-grade glioma; $\mathrm{MD}=$ mean diffusivity

G iomas are the most common type of WM-involved invasive cerebral primary neoplasm in adults. These brain tumors represent approximately $80 \%$ of primary malignant brain tumors and almost $3 \%$ of all types of cancer, and patient prognosis is poor.

In recent years, the use of noninvasive study techniques, such as cortical mapping and fMRI, has improved presurgical planning

Received December 30, 2014; accepted after revision March 10, 2015.

From the Departments of Biomedical Science and Morphological and Functional Images (E.M., F.G., A.A., M.G., S.L.V., M.L.) and Neurosciences (C.A., F.T., A.C.), University of Messina, Messina, Italy; and Scientific Institute for Recovery and Care Centro Neurolesi Bonino Pulejo (S.M.), Messina, Italy.

Please address correspondence to A. Arrigo, MD, Department of Biomedical Science and Morphological and Functional Images, University of Messina, via Consolare Valeria, one c/o A.O.U. Policlinico "G. Martino", 98125 Messina, Italy; e-mail: alessandro.arrigo@hotmail.com

-- Indicates open access to non-subscribers at www.ajnr.org

http://dx.doi.org/10.3174/ajnr.A4368 for brain neoplasms. However, these methods alone are considered inadequate to achieve the primary neurosurgical aim, obtaining the most radical tumor resection with the minimum of postoperative deficits, because they do not provide good anatomic representation of the spatial location of WM tracts affected by the tumor. ${ }^{1}$ Tractography is the most common neuroimaging technique used to reveal WM structure by analysis of DWI signals dependent on anisotropic water diffusion. ${ }^{2}$ From DWI gradient directions, it is possible to generate an anisotropic map showing WM bundles and their orientations; this information is adapted by tractographic algorithms to yield a 3D representation of WM tracts. DTI-based tractography is widely used for presurgical planning and is a powerful tool in the evaluation of major WM fiber bundles; it has also a positive impact on neurosurgical resection, disease prognosis, and preservation of brain function. ${ }^{3} \mathrm{Al}-$ though widely used and histologically validated, ${ }^{4}$ DTI approaches have several limitations, such as partial volume effects or lack of 
tensor estimation in voxels characterized by low fractional anisotropy (FA) values. ${ }^{5}$ Recent tractographic algorithms, such as probabilistic constrained spherical deconvolution (CSD), have overcome these limitations. ${ }^{6}$

The corticospinal tract (CST) and arcuate fasciculus (AF) are 2 of the WM pathways most commonly investigated by tractography because of their important roles in voluntary movement control and language, respectively. ${ }^{7}$ Probabilistic CSD improves tractographic reconstruction of the lateral portion of the CST, corresponding to the somatotopic representation of hand, face, tongue, and voluntary swallow muscles, which is not detectable by DTI-based approaches. ${ }^{8}$ In addition, this technique allows better evaluation of all AF components, including projections to the Geschwind area and other cortical regions, compared with other tractographic methods. ${ }^{9}$ The main aim of this study was to evaluate WM tract alterations of both the CST and AF in patients with frontoparietal high-grade gliomas (HGGs), through a qualitative and quantitative analysis by using probabilistic CSD tractography, to obtain reliable presurgical planning.

\section{MATERIALS AND METHODS Participants}

In our study, we recruited 20 patients ( 9 women and 11 men; mean age, $47.4 \pm 14.2$ years; age range, $20-67$ years), all affected by HGG, which involved mainly the lateral part of the frontoparietal lobes. After MR imaging evaluation, all patients underwent surgery, and all diagnoses were confirmed histologically.

The study was approved by our institution review board, and written informed consent was obtained from all subjects.

\section{Data Acquisition and Preprocessing}

The study was performed with an Achieva 3T MR imaging scanner (Philips Healthcare, Best, the Netherlands) by using a 32channel coil. We performed the following sequences:

- A T1-weighted fast-field echo 3D high-resolution sequence with TR, $8.1 \mathrm{~ms}$; TE, $3.7 \mathrm{~ms}$; flip angle, $8^{\circ}$; reconstruction matrix, $240 \times 240$; voxel size, $1 \mathrm{~mm}^{3}$ without an intersection gap;

- A FLAIR volume isotropic turbo spin-echo acquisition sensitivity encoding 3D sequence with TR, $8000 \mathrm{~ms}$; TE, $360 \mathrm{~ms}$; TI, $2400 \mathrm{~ms}$; reconstruction matrix, $240 \times 240$; voxel size, $1 \mathrm{~mm}^{3}$ without an intersection gap;

- Diffusion-weighted MR imaging acquired with a dual-phase encoded pulsed gradient spin-echo sequence with TR, 15,120 ms; TE, 54 ms; scan matrix, $160 \times 160$; section thickness, $2 \mathrm{~mm}$ without an intersection gap; 60 gradient directions; b-value, $1000 \mathrm{~s} / \mathrm{mm}^{2}$. We corrected the diffusion-weighted dataset for eddy current distortions and motion artifacts and adjusted the diffusion gradients with proper rotation of the b-matrix. ${ }^{10}$

\section{Data Processing and Fiber Tracking}

To avoid possible coregistration errors between morphologic and diffusion images caused by HGGs, ${ }^{8}$ we did not normalize patient data to a common template space as usual. Seed ROIs were also selected directly in the native space of each subject. Fiber tracking was obtained with a probabilistic CSD algorithm (MRtrix software package; http://software.incf.org/software/mrtrix/mrtrixpackage $)^{9}$ with manually selected ROIs. Fiber tracking was stopped when 10,000 tracts reached the selected ROI or when 100,000 total tracts were generated. The 3D segmentation of each HGG and the 3D visualization of tracts were performed by using 3D Slicer software (www.slicer.org) ${ }^{11}$ with T1-weighted images set as an overlay.

\section{Qualitative Analysis}

Qualitative analysis was performed by a radiologist with 20 years of experience who evaluated reconstructed tracts on superimposed 3D T1-weighted images. We verified the anatomic course of each fiber bundle section by section, in axial, coronal, and sagittal planes, comparing the neoplasm-affected side with the healthy one. During this step, the reader could detect any macroscopic WM tract dislocation or disruption in the affected side. Moreover, neoplasm 3D segmentation was used to depict the spatial relationship between tracts and gliomas.

\section{Quantitative and Statistical Analysis}

Quantitative analysis of reconstructed tracts, both in healthy and affected hemispheres, was performed by using the output data of MRtrix. Diffusion tensors were sampled along fiber tracts, and the mean values of FA, mean diffusivity (MD), linear coefficient $(\mathrm{Cl})$, planar coefficient $(\mathrm{Cp})$, and spherical coefficient (Cs) were considered for each bundle. All parameters were calculated with in-house scripts built with the Matlab software package (MathWorks, Natick, Massachusetts), based on the eigenvalues obtained from MRtrix output data.

As previously described, ${ }^{12-14}$ we used a combined approach of diffusion tensor parameters and probabilistic CSD tractography to overcome the well-known limitations of DTI in voxels containing crossing fibers and to increase the sensitivity of diffusion tensor metric changes in these regions.

Statistical analysis was performed by the nonparametric Wilcoxon signed rank test for paired measures of bundles in the healthy-versus-affected sides. Furthermore, Šidák correction was performed to account for multiple comparisons. The significance threshold was set to an $\alpha$ value of .05, resulting in an effective threshold of .0102 after correction.

\section{RESULTS}

All recruited patients were symptomatic with different degrees of motor and/or speech impairment, from mild to severe. All patients had both CST and AF involved by HGGs, which were located in the lateral frontoparietal lobes. There was a prevalence of left-sided involvement ( 11 left side, 9 right side). After surgery, the histologic analysis of resected neoplasms confirmed each lesion as III or IV World Health Organization grade: grade III $(n=15)$ and grade IV $(n=5)$. We were able to obtain tractographic reconstructions of the medial portion of the CST and the lateral portion of the CST and AF, both in the healthy and neoplasm-affected sides in all 20 patients.

\section{Qualitative Analysis}

Qualitative analysis revealed the anatomic course of each reconstructed fiber bundle, morphologic alterations of WM tracts on affected sides, and the spatial relationship between those tracts and the HGG. 

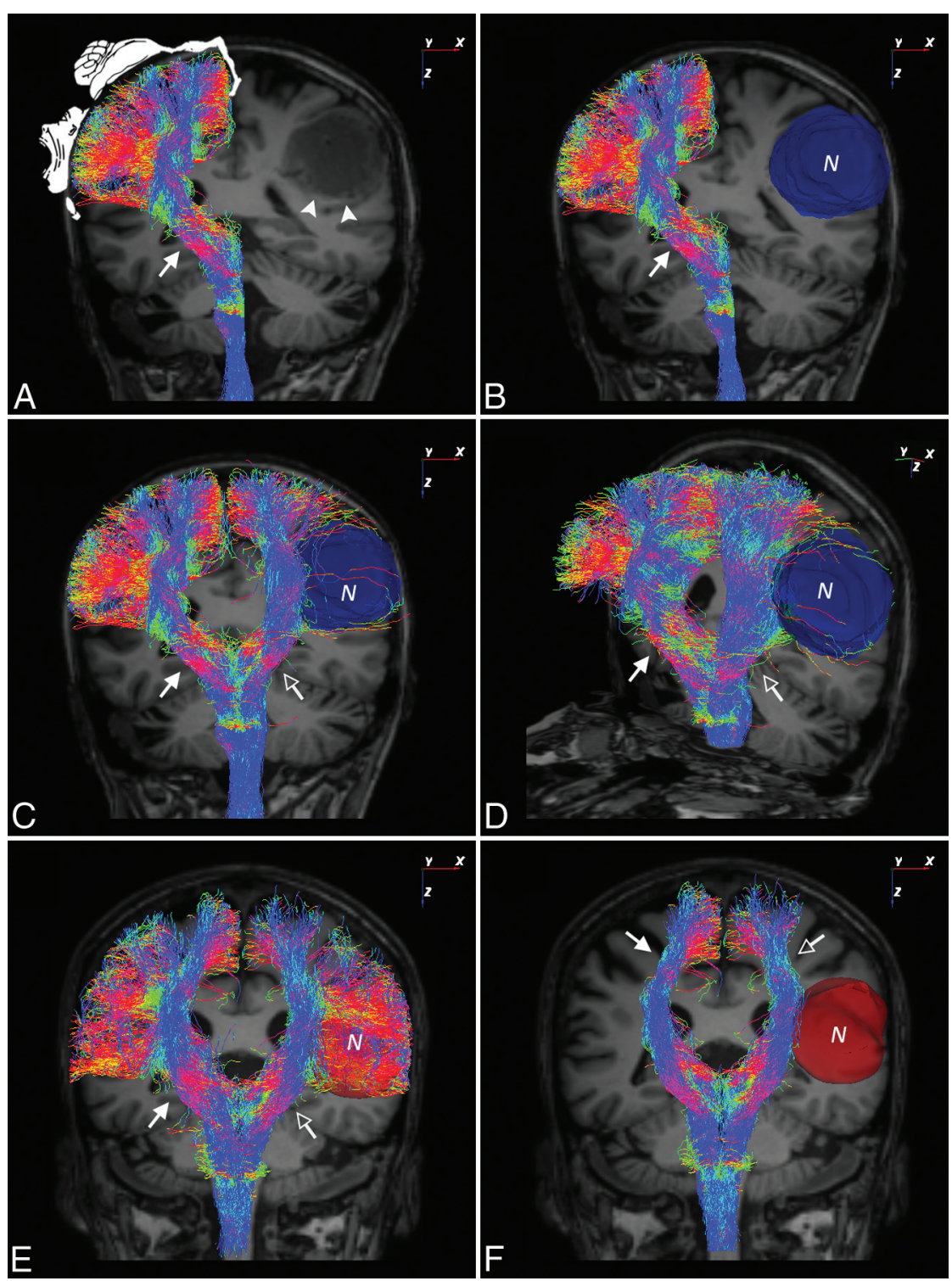

FIG 1. Tractographic reconstruction of corticospinal tracts in patients with high-grade gliomas. Coronal T1-weighted MR image (A) shows the corticospinal tract (arrow) on the healthy side with overlay of the Pensfield motor homunculus (arrowheads indicate neoplasm margins) and the neoplasm volume segmentation $(B)$ (blue volume). Coronal $(C)$ and sagittal $(D)$ rotated images show tractographic reconstruction of CSTs on both healthy (arrow) and neoplasm-affected sides (empty arrow) of the brain. In this patient, the lateral portion of the CST on the affected side is poorly represented due to neoplasm disruption. E, Coronal T1-weighted MR image shows CSTs, both on healthy (arrow) and neoplasm-affected sides (empty arrow) of another patient (neoplasm segmentation is depicted by red volume). In this case, on the affected side, the lateral portion of the CST passes through the neoplasm and is poorly involved. $F$, Coronal T1-weighted MR image shows the medial portion of CSTs on the healthy side (arrow) and the absence of their involvement in the neoplasm-affected one (empty arrow). $N$ indicates neoplasm.

Probabilistic CSD tractography of the CST yielded streamlines of both the medial and lateral portions of the CST on each side of the brain. Qualitative analysis for the healthy side showed a reliable representation of all components of the Pensfield homunculus (Fig $1 A$ ). In the neoplasm-affected side, all evaluated tracts showed a strict spatial relationship with HGGs. In 6/20 patients, the lateral portion of the CST was disrupted by the neoplasm, with consequent poor representation of streamlines (Fig $1 B-D$ ). In the other 14 patients, the lateral portion of the CST passed through the neoplasm and was, therefore, less compromised than expected on the basis of morphologic MR imaging evaluation of the neoplasms (Fig $1 E)$. In all patients, the medial portion of the CST was not affected by the neoplasm (Fig $1 F$ ).

Probabilistic CSD tractographic reconstructions also allowed reliable recognition of the anatomic course for all AF segments, such as Broca, Wernicke, and Geschwind projections (Fig 2) on both sides. Qualitative analysis showed that the AF was involved by the neoplasm on the affected side of all subjects (Fig $2 D-F$ ), with different degrees of morphologic impairment.

\section{Quantitative Analysis}

Quantitative analysis showed that FA was significantly decreased in the lateral portion of the CST $(z=2.51, n=20$, $P=.006)$ and in the $\operatorname{AF}(z=2.52, n=$ $20, P=.006)$ of the side affected by the neoplasm; furthermore, no significant difference was found for FA $(P>.0102)$ in the medial portion of the CST. Although MD was increased in all affected tracts, only the lateral portion of the CST reached significance $(z=-2.53, n=20$, $P=.006)$. Cl was significantly decreased in the affected bundles for the lateral portion of the CST $(z=2.51, n=20$, $P=.006)$ and the $\operatorname{AF}(z=2.52, n=20$, $P=.006)$. Cs was significantly increased for the affected lateral CST $(z=-2.51$, $n=20, P=.006)$ and for the $\operatorname{AF}(z=$ $-2.52, n=20, P=.006)$. No differences were observed when measuring Cp. Quantitative results are summarized in Fig 3.

\section{DISCUSSION}

In this study we evaluated 20 patients with HGGs centered in the lateral frontoparietal lobes. Each patient underwent glioma surgical resection after presurgical evaluation of the main WM fiber bundles involved by MR imaging tractography. Because the main goal of the surgical approach was to remove neoplasms while preserving brain function, accurate presurgical planning was essential to granting the best possible quality of life after surgery.

Presurgical tractographic analysis, performed by a probabilistic CSD-based tractographic approach, revealed involvement of the CST and AF in each patient. These $2 \mathrm{WM}$ fiber bundles have clinically relevant roles in voluntary motion control and speech and language, respectively. Common DTI approaches cannot depict the entire motor tract, allowing only reconstruction of the 

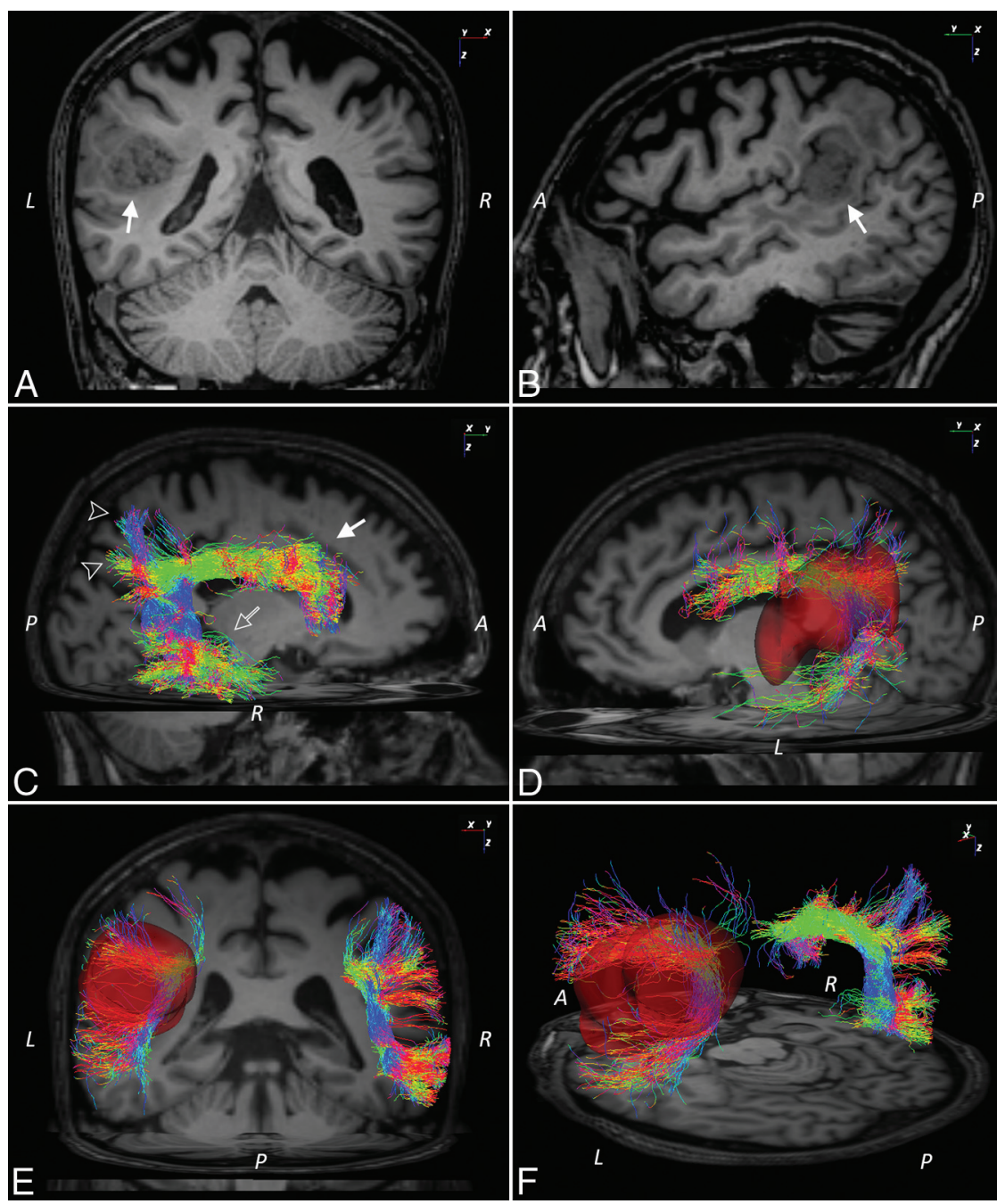

FIG 2. Tractographic reconstruction of arcuate fasciculi in a patient with a high-grade glioma. Coronal $(A)$ and sagittal (B) T1-weighted MR images show a frontoparietal high-grade glioma on the left side (arrow). C, Sagittal T1-weighted MR image shows tractographic reconstruction of the right arcuate fasciculus on the healthy side with the depiction of Broca (arrow), Wernicke (empty arrow), and Geschwind projections (empty arrowheads). D, The left AF on the affected side passes through the neoplasm (neoplasm segmentation is depicted by a red volume). Coronal (E) and axial $(F)$ tilted T1-weighted MR images show the AFs on both affected and healthy sides (neoplasm segmentation is depicted by a red volume). A indicates anterior; P, posterior; R, right; L, left.

medial portion of the CST, corresponding to a somatotopic depiction of the lower limbs, trunk, and upper limbs without the hand. Conversely, these techniques could not represent the lateral portion of the CST, which corresponds to a somatotopic depiction of the hand, face, tongue, and voluntary swallow muscles, ${ }^{15}$ due to the inherent limitations of DTI approaches to resolve complex fiber configurations, ${ }^{6}$ which were estimated to represent approximately $90 \%$ of WM voxels of the entire brain. ${ }^{16}$ By overcoming these limitations, probabilistic CSD-based tractography allowed a reconstruction of the entire CST (medial and lateral parts) ${ }^{8}$ with a marked match between tracts and all somatotopic parts of the Penfield motor homunculus. It also demonstrated that brain neoplasms can involve different WM modifications, resulting in DTI parameter changes and alterations of the average WM fiber bundle morphology represented with tractography. In particular, Witwer et $\mathrm{al}^{17}$ described deviated, infiltrated, edema- tous, and disrupted WM patterns, depending on tumor type and location.

Different DTI-based studies reported that high-grade gliomas mainly cause complete tract disruption, ${ }^{18}$ whereas low-grade gliomas infiltrate tracts along myelinated fibers. ${ }^{19}$ All these patterns of tumors cause DTI parameter changes, in particular FA decrease, in involved regions. ${ }^{20}$ Furthermore, the presence of voxels with FA values lower than the DTI threshold (commonly set to 0.2 ) causes an interruption of reconstructed WM tracts, ${ }^{21}$ and the use of an FA threshold lower than 0.2 results in poor accuracy of major eigenvector-direction estimation. ${ }^{1}$ These limitations can produce 3 negative effects on tractographic presurgical planning, particularly in CST evaluation. First, the reconstructed CST tract could show a false interruption, for example, in the lateral portion of the $\mathrm{CST}^{20}{ }^{20}$ Second, DTI findings could suggest a false safe resection margin around the lesion. ${ }^{8}$ Last, the lack of reconstruction of the lateral portion of the CST fails to provide any qualitative or quantitative information about this part of the tract and its relationship to neoplasms. These combined effects make presurgical planning inaccurate and may contribute to unexpected postoperative functional deficits.

Use of the CSD technique allowed reconstruction of fibers in voxels with low anisotropy and characterization of voxels in tumoral and peritumoral areas ${ }^{1}$ and voxels with complex axonal spatial configurations. In addition, it was demonstrated that edema-affected and infiltrated tracts could reduce their anisot- ropy, while preserving sufficient directional information for tractographic depiction. ${ }^{1}$

In our study, we could reconstruct the medial and lateral portions of the CST of 20 patients in both the healthy and neoplasmaffected sides of the brain by using probabilistic CSD tractography. This approach allowed reliable reconstruction of these pathways with an accurate representation of the entire Penfield motor homunculus on the healthy side, avoiding well-known reconstruction problems from crossing fibers. Moreover, on the involved side, we detected differing degrees of involvement for the lateral portion of the CST, from deviation to disruption, with no alterations of the medial portion.

AF depiction in presurgical planning is clinically relevant to avoid aphasic syndromes induced by surgical lesions or stroke. $^{21,22}$ This WM bundle connects to Broca, Wernicke, and Geschwind areas and other brain regions involved in language 

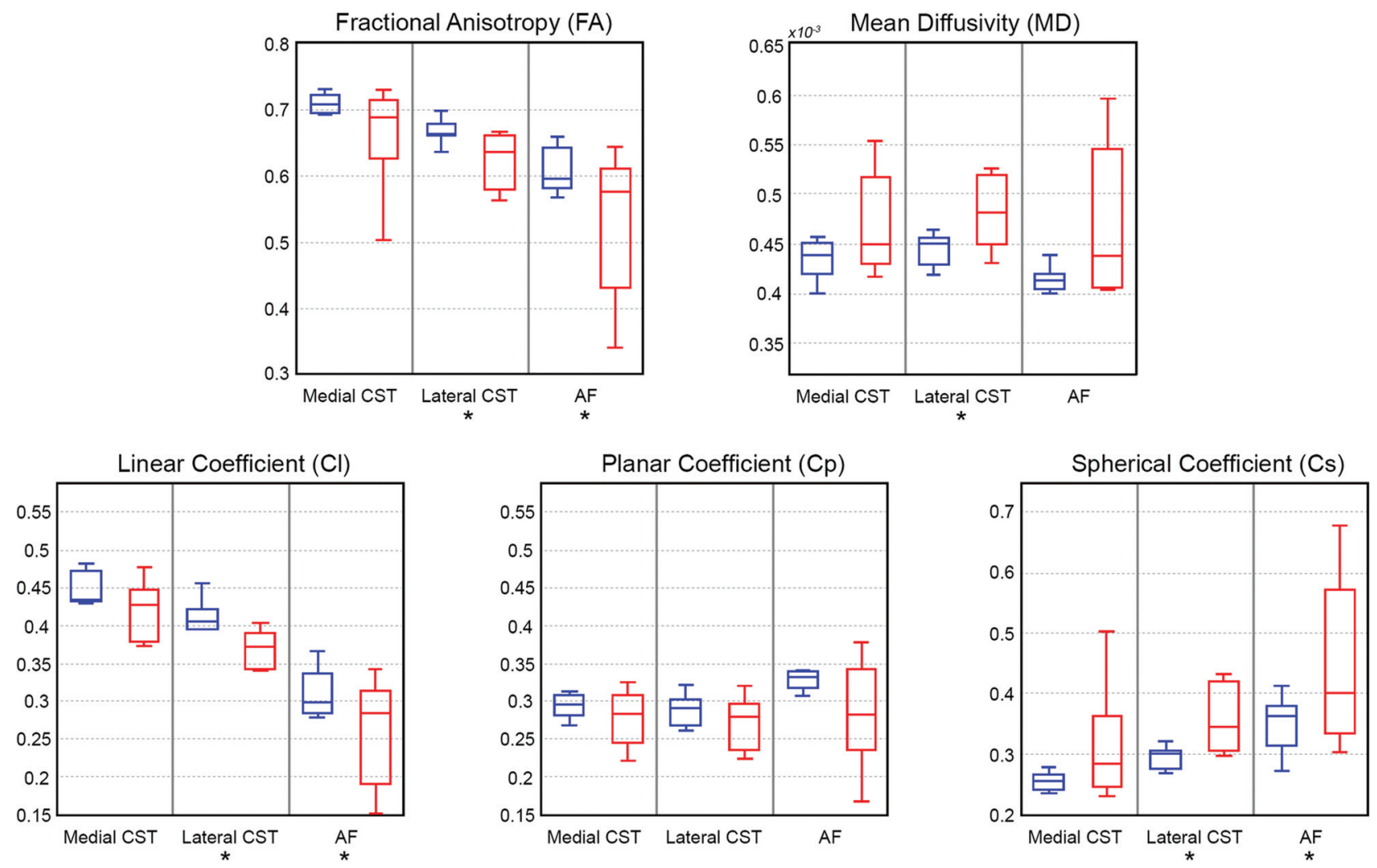

FIG 3. Diffusion tensor parameter distributions of the medial and lateral corticospinal portions and arcuate fasciculi. Blue and red boxplots represent DTI parameter distributions in the healthy and neoplasm-affected sides, respectively. Asterisks indicate statistical significance $(P<.05$ corrected for multiple comparisons).

and speech. ${ }^{23}$ Because brain stimulation techniques are less effective for WM tracts than for GM, evaluating the morphologic localization of the AF is essential for appropriate presurgical planning. ${ }^{24-27}$ DTI-based tractographic studies revealed incomplete AF reconstructions, in particular of the anterior portion. ${ }^{24}$ The evaluation of this AF segment is considered an important predictor of postoperative outcome because a lesion in the anterior portion of AF could cause negative effects on speech fluency. ${ }^{28}$ DTI approaches have tractographic reconstruction limitations in the centrum semiovale, due to the presence of crossing fibers. ${ }^{24}$ In addition, DTI tractography of AFs in patients with brain neoplasms resulted in incomplete correspondence with intracortical stimulation, suggesting that this technique is not optimal for mapping language areas. ${ }^{29}$ Finally, the same negative effects of DTI techniques discussed above for CST are also relevant to AFs involved by gliomas during presurgical planning. Thus, the use of probabilistic CSD, which includes voxels not considered by DTI tractography, could provide advantages in the evaluation of DTI metrics in aphasic syndromes as well.

In this cohort of patients, we found that probabilistic CSDbased tractography allowed reliable representation of AFs both in glioma-involved and noninvolved sides of the brain, even in arduous regions for conventional DTI tractography. Qualitative analysis showed that all affected AFs were dislocated, infiltrated, or disrupted by the neoplasm.

From probabilistic CSD tractography, we extrapolated a quantitative analysis based on the main DTI parameters (FA, MD, Cp, $\mathrm{Cl}$, and $\mathrm{Cs}$ ). FA and MD are well-known values measuring axonal integrity and anisotropic water diffusivity, respectively. $\mathrm{Cl}$ measures the intravoxel uniformity of tract direction and fiber tract organization, Cs estimates the intravoxel diffusivity, ${ }^{30}$ and Cp estimates voxels in which there are crossing or kissing fibers. ${ }^{12}$ No statistically significant changes were found for the medial CST both in healthy and affected sides of the brain, suggesting that in our group of patients, these bundles are not involved with HGGs. In all patients, we found statistically significant differences in both the lateral CST and AF between the involved and noninvolved sides. In particular, we detected decreased FA and $\mathrm{Cl}$ and increased Cs in the involved side. MD was increased in all affected tracts, reaching significance only in the lateral portion of CST, probably due to the low number of subjects. The significant FA decrease could reflect a remarkable change in WM microstructure, induced by tract deviation, infiltration, or disruption or influenced by edema. ${ }^{30} \mathrm{MD}$ increase could be associated with loss of WM integrity with a consequent increase in free tissue water. ${ }^{31} \mathrm{Cl}$ and Cs are 2 known shape-oriented anisotropy measures, indexes of anisotropic and isotropic diffusion changes, respectively. ${ }^{30}$ In our study, neoplasms changed the intravoxel uniformity and diffusivity of affected tracts, causing $\mathrm{Cl}$ decrease and Cs increase. These parameters, combined with the use of FA and MD, could provide a powerful quantitative estimation of WM tracts involved by HGGs. Finally, we evaluated Cp for all tracts, which is another shape-oriented parameter reflecting the intravoxel presence of crossing fibers. ${ }^{30}$ In our study, the Cp value was not significantly different between the healthy and affected side. This result could 
be due to a lack of sensitivity in cases of $>2$ crossing fibers inside the same voxel. ${ }^{12}$

The major limitation of this study is the relatively small patient cohort, which might influence the statistical power of our findings. In addition, it was not possible to perform follow-up MR imaging tractography after surgical neoplasm resections. This lack of MR imaging follow-up prohibited us from providing an evaluation of the postoperative outcome after CSD-based presurgery planning.

\section{CONCLUSIONS}

The results presented here demonstrated that probabilistic CSD tractography provides useful qualitative and quantitative analysis in presurgical planning for HGGs. Our qualitative analysis showed that probabilistic CSD allowed reliable reconstruction of tracts not detected with other DTI techniques, such as those involved by neoplasms or with complex fiber configurations. We also demonstrated that quantitative analysis based on CSD tractography can characterize the involvement of the tracts by the neoplasms, overcoming the well-known quantitative underestimation related to DTI reconstruction. Furthermore, because postoperative quantitative measurements are also important for the prediction of brain-function recovery, ${ }^{32}$ further studies performed with probabilistic CSD could provide noteworthy results on surgical-outcome evaluation.

\section{REFERENCES}

1. Jellison BJ, Field AS, Medow J, et al. Diffusion tensor imaging of cerebral white matter: a pictorial review of physics, fiber tract anatomy, and tumor imaging patterns. AJNR Am J Neuroradiol 2004; 25:356-69

2. Basser PJ, Pajevic S, Pierpaoli C, et al. In vivo fiber tractography using DT-MRI data. Magn Reson Med 2000;44:625-32

3. Romano A, D'Andrea G, Minniti G, et al. Pre-surgical planning and MR-tractography utility in brain tumour resection. Eur Radiol 2009; 19:2798-808

4. Dyrby TB, Søgaard LV, Parker GJ, et al. Validation of in vitro probabilistic tractography. Neuroimage 2007;37:1267-77

5. Jones DK, Cercignani M. Twenty-five pitfalls in the analysis of diffusion MRI data. NMR Biomed 2010;23:803-20

6. Tournier JD, Calamante F, Connelly A. Robust determination of the fibre orientation distribution in diffusion MRI: non-negativity constrained super-resolved spherical deconvolution. Neuroimage 2007;35:1459-72

7. Kristo G, Leemans A, Raemaekers M, et al. Reliability of two clinically relevant fiber pathways reconstructed with constrained spherical deconvolution. Magn Reson Med 2013;70:1544-56

8. Farquharson S, Tournier JD, Calamante F, et al. White matter fiber tractography: why we need to move beyond DTI. J Neurosurg 2013;118:1367-77

9. Tournier JD, Calamante F, Connelly A. MRtrix: diffusion tractography in crossing fiber regions. International Journal of Imaging Systems and Technology 2012;22:53-66

10. Leemans A, Jones DK. The B-matrix must be rotated when correcting for subject motion in DTI data. Magn Reson Med 2009: 61:1336-49

11. Fedorov A, Beichel R, Kalpathy-Cramer J, et al. 3D Slicer as an image computing platform for the Quantitative Imaging Network. Magn Reson Imaging 2012;30:1323-41

12. Reijmer YD, Leemans A, Heringa SM, et al. Improved sensitivity to cerebral white matter abnormalities in Alzheimer's disease with spherical deconvolution based tractography. PLoS One 2012; 7:e44074
13. Arrigo A, Mormina E, Anastasi GP, et al. Constrained spherical deconvolution analysis of the limbic network in human, with emphasis on a direct cerebello-limbic pathway. Front Hum Neurosci 2014;8:987

14. Mormina E, Arrigo A, Calamuneri A, et al. Diffusion tensor imaging parameters' changes of cerebellar hemispheres in Parkinson's disease. Neuroradiology 2015;57:327-34

15. Kinoshita M, Yamada K, Hashimoto N, et al. Fiber-tracking does not accurately estimate size of fiber bundle in pathological condition: initial neurosurgical experience using neuronavigation and subcortical white matter stimulation. Neuroimage 2005;25:424-29

16. Jeurissen B, Leemans A, Tournier JD, et al. Investigating the prevalence of complex fiber configurations in white matter tissue with diffusion magnetic resonance imaging. Hum Brain Mapp 2013: 34:2747-66

17. Witwer BP, Moftakhar R, Hasan KM, et al. Diffusion-tensor imaging of white matter tracts in patients with cerebral neoplasm. J Neurosurg 2002;97:568-75

18. Chen F, Zhang X, Li M, et al. Axial diffusivity and tensor shape as early markers to assess cerebral white matter damage caused by brain tumors using quantitative diffusion tensor tractography. CNS Neurosci Ther 2012;18:667-73

19. Duffau H, Khalil I, Gatignol P, et al. Surgical removal of corpus callosum infiltrated by low-grade glioma: functional outcome and oncological considerations. J Neurosurg 2004;100:431-37

20. Min ZG, Rana N, Niu C, et al. Does diffusion tensor tractography of the corticospinal tract correctly reflect motor function? Med Princ Pract 2014;23:174-76

21. Kim SH, Jang SH. Prediction of aphasia outcome using diffusion tensor tractography for arcuate fasciculus in stroke. AJNR Am J Neuroradiol 2013;34:785-90

22. Hayashi Y, Kinoshita M, Nakada M, et al. Correlation between language function and the left arcuate fasciculus detected by diffusion tensor imaging tractography after brain tumor surgery. J Neurosurg 2012;117:839-43

23. Henning Stieglitz L, Seidel K, Wiest R, et al. Localization of primary language areas by arcuate fascicle fiber tracking. Neurosurgery 2012; 70:56-64; discussion 64-65

24. Li Z, Peck KK, Brennan NP, et al. Diffusion tensor tractography of the arcuate fasciculus in patients with brain tumors: comparison between deterministic and probabilistic models. J Biomed Sci Eng 2013;6:192-200

25. Roux FE, Boulanouar K, Ranjeva JP, et al. Cortical intraoperative stimulation in brain tumors as a tool to evaluate spatial data from motor functional MRI. Invest Radiol 1999;34:225-29

26. Cosgrove GR, Buchbinder BR, Jiang H. Functional magnetic resonance imaging for intracranial navigation. Neurosurg Clin $\mathrm{N} \mathrm{Am}$ 1996;7:313-22

27. Mueller WM, Yetkin FZ, Hammeke TA, et al. Functional magnetic resonance imaging mapping of the motor cortex in patients with cerebral tumors. Neurosurgery 1996;39:515-20; discussion 520-21

28. Fridriksson J, Guo D, Fillmore P, et al. Damage to the anterior arcuate fasciculus predicts non-fluent speech production in aphasia. Brain 2013;136:3451-60

29. Leclercq D, Duffau H, Delmaire C, et al. Comparison of diffusion tensor imaging tractography of language tracts and intraoperative subcortical stimulations. J Neurosurg 2010;112:503-11

30. Westin CF, Maier SE, Mamata $\mathrm{H}$, et al. Processing and visualization for diffusion tensor MRI. Med Image Anal 2002;6:93-108

31. Sternberg EJ, Lipton ML, Burns J. Utility of diffusion tensor imaging in evaluation of the peritumoral region in patients with primary and metastatic brain tumors. AJNR Am J Neuroradiol 2014; 35:439-44

32. Kinoshita M, Nakada M, Okita $\mathrm{H}$, et al. Predictive value of fractional anisotropy of the arcuate fasciculus for the functional recovery of language after brain tumor resection: a preliminary study. Clin Neurol Neurosurg 2014;117:45-50 